\title{
MORE-OR-LESS-UNIFORM SAMPLING AND LENGTHS OF CURVES
}

\author{
BY
}

LYLE NOAKES (Department of Mathematics and Statistics, The University of Western Australia, 35 Stirling Highway, Crawley WA 6009, Australia)

AND

RYSZARD KOZERA (Department of Computer Science and Software Engineering, The University of Western Australia, 35 Stirling Highway, Crawley WA 6009, Australia)

Abstract. More-or-less-uniform samples are introduced and used to estimate lengths of smooth regular strictly convex curves in $\mathbb{R}^{2}$. Quartic convergence is proved and illustrated by examples.

1. Introduction. The problem of measuring the length of a curve has a long history in mathematics, dating back to ancient geometry. In particular, Archimedes and Liu Hui [11] estimated the length of a circular curve. Jordan, Peano and others introduced digitizations of sets in $\mathbb{R}^{2}$ and $\mathbb{R}^{3}$ for the purpose of different feature measurements such as perimeters (see, e.g., [4]). Related historical and contemporary work can be found in $[2],[3],[6],[7],[9],[10]$, and [12].

Let $\gamma:[0, T] \rightarrow \mathbb{R}^{n}$ be a smooth regular curve; namely, $\gamma$ is $C^{k}$ for some $k \geq 1$ and $\dot{\gamma}(t) \neq \mathbf{0}$ for all $t \in[0, T]$. The length of $\gamma$ is defined to be

$$
d(\gamma)=\int_{0}^{T}\|\dot{\gamma}(t)\| d t,
$$

where $\dot{\gamma}$ is the derivative of $\gamma$, and $\|\cdot\|$ is the Euclidean norm. Consider the problem of estimating $d(\gamma)$ from an ordered $(m+1)$-tuple

$$
\mathcal{Q}=\left(q_{0}, q_{1}, \ldots, q_{m}\right)
$$

of points in $\mathbb{R}^{n}$, where $q_{i}=\gamma\left(t_{i}\right)$, and $0=t_{0}<t_{1}<\cdots<t_{i}<\cdots<t_{m}=T$. Depending on what is known about the $t_{i}$, the problem may be straightforward or unsolvable.

EXAMPLE 1. Let $\gamma$ be $C^{r+2}$, where $r$ is a positive integer, and take $m$ to be a multiple of $r$. Then $\mathcal{Q}$ gives $\frac{m}{r}(r+1)$-tuples of the form

$$
\left(q_{0}, q_{1}, \ldots, q_{r}\right), \quad\left(q_{r}, q_{r+1}, \ldots, q_{2 r}\right), \ldots \quad,\left(q_{m-r}, q_{m-r+1}, \ldots, q_{m}\right) .
$$

Received April 10, 2001.

2000 Mathematics Subject Classification. Primary 65Dxx.

E-mail address: lyle@maths.uwa.edu.au

E-mail address: ryszard@cs.uwa.edu.au 
The $j$ th $(r+1)$-tuple can be interpolated by a polynomial $\hat{\gamma}_{j}:\left[t_{(j-1) r}, t_{j r}\right] \rightarrow \mathbb{R}^{n}$ of degree $r$, and the track-sum $\hat{\gamma}$ of the $\hat{\gamma}_{j}$ is everywhere continuous and $C^{\infty}$ except at the knot points $t_{r}, t_{2 r}, \ldots, t_{m-r}$. Suppose that sampling is uniform: $t_{i}=\frac{i T}{m}$ for $0 \leq i \leq m$. The errors in Lagrange interpolation are best studied using Lemma 2.1 in Sec. 2 of Part I of [5]. We find that $\hat{\gamma}(t)=\gamma(t)+O\left(\frac{1}{m^{r+1}}\right)$ for $t \in[0, T]$, and $\dot{\hat{\gamma}}=\dot{\gamma}(t)+O\left(\frac{1}{m^{r}}\right)$ for $t \neq t_{r}, t_{2 r}, \ldots, t_{m-r}$. Consequently, $d(\hat{\gamma})-d(\gamma)=O\left(\frac{1}{m^{r}}\right)$. This error can be shown to be $O\left(\frac{1}{m^{r+2}}\right)$ or $O\left(\frac{1}{m^{r+1}}\right)$ according as $r$ is even or odd [8].

EXAMPLE 2. Let $t_{1}=\frac{T}{2}$ and $t_{i}=t_{1}+\frac{i T}{2 m}$ for $2 \leq i \leq m$. $\mathcal{Q}$ gives only endpoint information for $\gamma$ over $\left[0, \frac{T}{2}\right]$, and therefore does not even determine an upper bound on $d(\gamma)$ as $m \rightarrow \infty$

An intermediate situation is where the $t_{i}$ are not given, but sampled more-or-less uniformly in the following sense.

Definition 1. Sampling is more-or-less uniform when there are constants $0<K_{l}<$ $K_{u}$ such that, for any sufficiently large integer $m$, and any $1 \leq i \leq m$,

$$
\frac{K_{l}}{m} \leq t_{i}-t_{i-1} \leq \frac{K_{u}}{m}
$$

The uniform sampling of Example 1 is more-or-less uniform, and the sampling in Example 2 is not. With more-or-less uniform sampling, increments between successive parameters are neither large nor small in proportion to $\frac{T}{m}$. Then, just as for the uniform sampling of Example 1, piecewise-linear interpolation between sample points approximates the image of $\gamma$ to $O\left(\frac{1}{m^{2}}\right)$, and $d(\gamma)$ to $O\left(\frac{1}{m^{2}}\right)$. However, use of piecewise-quadratic instead of piecewise-linear can lead to unfortunate results, because of the need to estimate $^{1}$ the parameters $t_{i}$ for $0 \leq i \leq m$. If we guess $t_{i}=\frac{i}{m}$, then the resulting piecewise-quadratic $\hat{\gamma}:[0,1] \rightarrow \mathbb{R}^{n}$ is sometimes informative [7], [8], and sometimes not.

EXAMPLE 3. For $0<i<m$, set $t_{i}=\frac{\left(3 i+(-1)^{i}\right) T}{3 m}$. Then sampling is more-or-less uniform, with $K_{l}=\frac{T}{3}, K_{u}=\frac{5 T}{3}$. Let $\gamma:[0, \pi] \rightarrow \mathbb{R}^{2}$ be the parametrization $\gamma(t)=$ $(\cos t, \sin t)$ of the unit semicircle in the upper half-plane. When $m$ is small, the image of $\hat{\gamma}$ does not much resemble a semicircle, as in Fig. 1 where $m=3$ and $d(\hat{\gamma})-d(\gamma)=0.0601$. The error in length estimate with piecewise-linear interpolation is -0.0712 . When $m$ is large the image of $\hat{\gamma}$ looks semicircular, as in Fig. 2 where $m=30$. In this case, however, $d(\hat{\gamma})-d(\gamma)=0.1194$, an error nearly twice as large as for $m=6$. Even piecewise-linear interpolation with 31 points gives a better estimate, with error -0.0033 . Indeed, as $m$ increases (at least for $m \leq 100$ ), piecewise-quadratic interpolation tends to increase errors of length estimates. Linear interpolation is better, but not impressive.

EXAMPLE 4. For $0<i<m$, let $t_{i}$ be a random number (according to some distribution) in the interval $\left[\frac{(3 i-1) T}{3 m}, \frac{(3 i+1) T}{3 m}\right]$. Then sampling is more-or-less uniform, with $K_{u}, K_{l}$ as in Example 3 .

Example 5. Choose $\theta>0$ and $0<L_{l}<L_{u}$. Set $s_{0}=0$. For $1 \leq i \leq m$, choose $\delta_{i} \in\left[\frac{L_{l}}{m}, \frac{L_{u}}{m}\right]$ independently from (say) the uniform distribution. Define $s_{i}=s_{i-1}+\delta_{i}$ for $i=1,2, \ldots, m$. The expectation of $s_{m}$ is $\frac{L_{u}+L_{l}}{2}$ and the standard deviation $\frac{L_{u}-L_{l}}{2 \sqrt{3 m}}$.

\footnotetext{
${ }^{1}$ In Example 1 these were assumed to be given.
} 


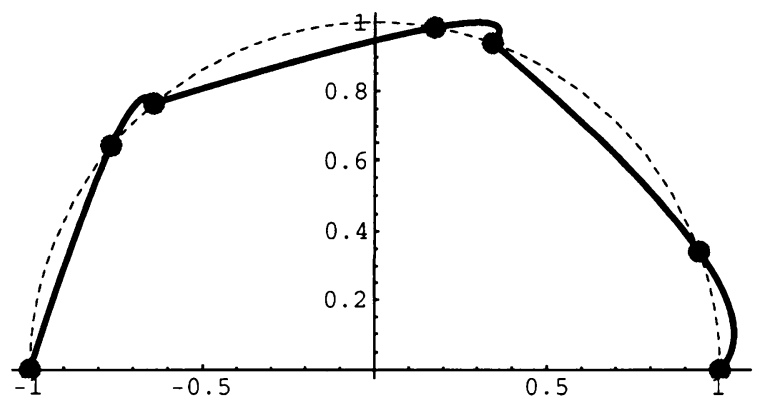

FIG. 1. 7 data points, with 3 successive triples interpolated by piecewise-quadratics, giving length estimate $\pi+0.0601035$ for the semicircle (shown dashed).

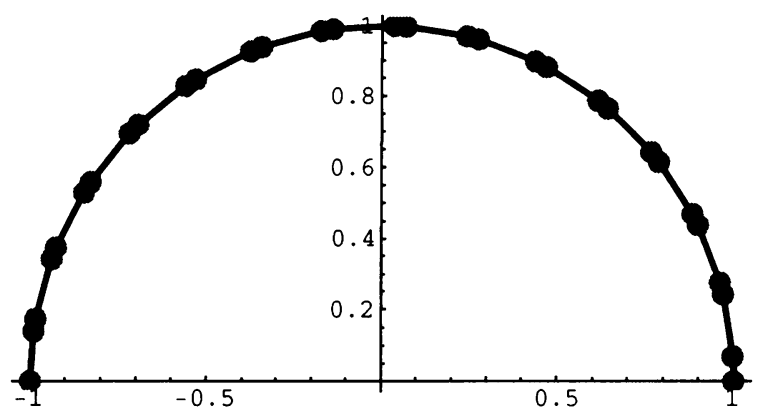

FIG. 2. 31 data points, with 15 successive triples interpolated by piecewise-quadratics, giving length estimate $\pi+0.119407$ for the semicircle.

So if $m$ is large, $s_{m} \approx \frac{L_{u}+L_{l}}{2}$ with high probability. For $0 \leq i \leq m$. define $t_{i}=\frac{s_{i} T}{s_{m}}$. Set

$$
K_{l}=\frac{2 L_{l} T}{L_{u}+L_{l}}-\theta, \quad K_{u}=\frac{2 L_{u} T}{L_{u}+L_{l}}+\theta .
$$

Then with high probability for $m$ large, the sampling $\left(t_{0}, t_{1}, t_{2}, \ldots, t_{m}\right)$ from $[0, T]$ is more-or-less uniform with constants $K_{l}, K_{u}$.

More-or-less uniform sampling is invariant with respect to reparameterizations; namely, if $\phi:[0, T] \rightarrow[0, T]$ is an order-preserving $C^{1}$ diffeomorphism, and if $\left(t_{0}, t_{1}, \ldots, t_{m}\right)$ are sampled more-or-less uniformly, then so are $\left(\phi\left(t_{0}\right), \phi\left(t_{1}\right), \ldots, \phi\left(t_{m}\right)\right)$. So reparameterizations lead to further examples from the ones already given. To state our main result, first take $n=2$ and suppose that $\gamma$ is $C^{4}$ and (without loss) parameterized by arc-length; namely, $\|\dot{\gamma}\|$ is identically 1 . The curvature of $\gamma$ is defined as

$$
k(t)=\operatorname{det}(M(t)),
$$

where $M(t)$ is the $2 \times 2$ matrix with columns $\dot{\gamma}(t), \ddot{\gamma}(t)$. When $k(t) \neq 0$ for all $t \in[0, T]$, $\gamma$ is said to be strictly convex. 
TheOREM 1. Let $\gamma:[0, T] \rightarrow \mathbb{R}^{2}$ be strictly convex and suppose that sampling is moreor-less uniform. Then, for some $\tilde{d}(\mathcal{Q})$, calculable in terms of $\mathcal{Q}$,

$$
\tilde{d}(\mathcal{Q})=d(\gamma)+O\left(\frac{1}{m^{4}}\right) .
$$

In Sections 2, 3 we prove Theorem 1, constructing $\tilde{d}(\mathcal{Q})$ as a sum of lengths of quadratic arcs interpolating quadruples of sample points. In Sec. 4, some examples are given, showing that the quartic convergence of Theorem 1 is the best possible for our construction.

Note added in proof. The authors have recently become aware of [13] which contains work on closely related problems and other interesting references.

2. Quadratics interpolating quadruples. Let $\mathcal{Q}$ be sampled more-or-less uniformly from $\gamma$, and suppose (without loss) that $m$ is a positive integer multiple of 3 . For each quadruple $\left(q_{i}, q_{i+1}, q_{i+2}, q_{i+3}\right)$, where $0 \leq i \leq m-3$, define $a_{0}, a_{1}, a_{2} \in \mathbb{R}^{2}$ and $Q^{i}(s)=a_{0}+a_{1} s+a_{2} s^{2}$ by

$$
Q^{i}(0)=q_{i}, \quad Q^{i}(1)=q_{i+1}, \quad Q^{i}(\alpha)=q_{i+2}, \quad \text { and } \quad Q^{i}(\beta)=q_{i+3} .
$$

Then $a_{0}=q_{i}, a_{2}=q_{i+1}-a_{0}-a_{1}$, and we obtain two vector equations:

$$
a_{1} \alpha+\left(p_{1}-a_{1}\right) \alpha^{2}=p_{\alpha} \quad \text { and } \quad a_{1} \beta+\left(p_{1}-a_{1}\right) \beta^{2}=p_{\beta}
$$

where $\left(p_{1}, p_{\alpha}, p_{\beta}\right) \equiv\left(q_{i+1}-q_{i}, q_{i+2}-q_{i}, q_{i+3}-q_{i}\right)$. Then (1) amounts to four quadratic scalar equations in four scalar unknowns $a_{1}=\left(a_{11}, a_{12}\right), \alpha, \beta$. Set

$$
c=-\operatorname{det}\left(p_{\alpha}, p_{\beta}\right), \quad d=-\operatorname{det}\left(p_{\beta}, p_{1}\right) / c, \quad e=-\operatorname{det}\left(p_{\alpha}, p_{1}\right) / c,
$$

where $c, d, e \neq 0$ by strict convexity, and define

$$
\rho_{1}=\sqrt{e(1+d-e) / d}, \quad \rho_{2}=\sqrt{d(1+d-e) / e} .
$$

Then (1) has two solutions (as can be verified by substitution):

$$
\left(\alpha_{+}, \beta_{+}\right)=\frac{\left(1+\rho_{1}, 1+\rho_{2}\right)}{e-d}, \quad\left(\alpha_{-}, \beta_{-}\right)=\frac{\left(1-\rho_{1}, 1-\rho_{2}\right)}{e-d},
$$

provided $\rho_{1}, \rho_{2}$ are real and $d-e \neq 0$. We now justify these assumptions and show that precisely one of (2) satisfies the additional condition

$$
1<\alpha<\beta \text {. }
$$

It suffices ${ }^{2}$ to deal with the case where $k(t)<0$ for all $t \in[0, T]$. Then it is rather apparent, for geometrical reasons, that $1+d-e,-d,-e$, and $e-d$ are all positive asymptotically. Alternatively, these facts can be proved (and sharper estimates obtained) by Mathematica calculations, as in Lemma 1 below. Define

$$
l(t)=\frac{\operatorname{det}\left(\frac{d \gamma}{d t}, \frac{d^{3} \gamma}{d t^{3}}\right)}{k(t)} .
$$

\footnotetext{
${ }^{2}$ The other case, where $k(t)$ is everywhere positive, is dealt with by considering the reversed curve $\gamma_{r}(t)=\left(\gamma_{1}(T-t), \gamma_{2}(T-t)\right)$.
} 
Then, using Taylor's theorem, for $t, u \in\left[t_{i}, t_{i+3}\right]$,

$$
\operatorname{det}\left(\gamma(t)-q_{i}, \gamma(u)-q_{i}\right)=k \frac{\left(t-t_{i}\right)\left(u-t_{i}\right)(u-t)}{2}\left(1+\left(u-2 t_{i}+t\right) \frac{l}{3}\right)+O\left(\frac{1}{m^{5}}\right)
$$

where $k, l$ are evaluated at $t_{i}$.

Lemma 1.

$$
\left(\alpha_{+}, \beta_{+}\right)=\frac{\left(\left(t_{i+2}-t_{i}\right)\left(1+\frac{l\left(t_{i+2}-t_{i+1}\right)}{6}\right),\left(t_{i+3}-t_{i}\right)\left(1+\frac{l\left(t_{i+3}-t_{i+1}\right)}{6}\right)\right)}{t_{i+1}-t_{i}}+O\left(\frac{1}{m^{2}}\right) .
$$

Proof. By (4),

$$
\begin{gathered}
c=-k \frac{\left(t_{i+2}-t_{i}\right)\left(t_{i+3}-t_{i}\right)\left(t_{i+3}-t_{i+2}\right)}{2}\left(1+\left(t_{i+3}-2 t_{i}+t_{i+2}\right) \frac{l}{3}\right)+O\left(\frac{1}{m^{5}}\right), \\
c d=-k \frac{\left(t_{i+3}-t_{i}\right)\left(t_{i+1}-t_{i}\right)\left(t_{i+1}-t_{i+3}\right)}{2}\left(1+\left(t_{i+1}-2 t_{i}+t_{i+3}\right) \frac{l}{3}\right)+O\left(\frac{1}{m^{5}}\right), \\
c e=-k \frac{\left(t_{i+2}-t_{i}\right)\left(t_{i+1}-t_{i}\right)\left(t_{i+1}-t_{i+2}\right)}{2}\left(1+\left(t_{i+1}-2 t_{i}+t_{i+2}\right) \frac{l}{3}\right)+O\left(\frac{1}{m^{5}}\right) .
\end{gathered}
$$

Consequently,

$$
\begin{aligned}
& -d=\frac{t_{i+1}-t_{i}}{t_{i+3}-t_{i+2}} \frac{t_{i+3}-t_{i+1}}{t_{i+2}-t_{i}}\left(1-\left(t_{i+2}-t_{i+1}\right) \frac{l}{3}\right)+O\left(\frac{1}{m^{2}}\right), \\
& -e=\frac{t_{i+1}-t_{i}}{t_{i+3}-t_{i+2}} \frac{t_{i+2}-t_{i+1}}{t_{i+3}-t_{i}}\left(1-\left(t_{i+3}-t_{i+1}\right) \frac{l}{3}\right)+O\left(\frac{1}{m^{2}}\right) .
\end{aligned}
$$

The lemma follows from these two equations. The detailed calculation can be viewed at the URL address http://www.cs.uwa.edu.au/ ryszard/4points/.

We continue with the assumption that $k(t)<0$ for all $t$. Then (3) follows from (5) for $m$ large with $(\alpha, \beta)=\left(\alpha_{+}, \beta_{+}\right)$. Then, for $0 \leq s \leq \beta, Q^{i}(s)=q_{i}+a_{1} s+a_{2} s^{2}$, where

$$
a_{1}=\frac{p_{\alpha}-\alpha^{2} p_{1}}{\alpha-\alpha^{2}}=\frac{p_{\beta}-\beta^{2} p_{1}}{\beta-\beta^{2}} \quad \text { and } \quad a_{2}=\frac{\alpha p_{1}-p_{\alpha}}{\alpha-\alpha^{2}}=\frac{\beta p_{1}-p_{\beta}}{\beta-\beta^{2}} .
$$

LEMMA 2.

$$
\begin{aligned}
& a_{1}=\left(t_{i+1}-t_{i}\right) \dot{\gamma}\left(t_{i}\right)\left(1+O\left(\frac{1}{m}\right)\right)+O\left(\frac{1}{m^{3}}\right), \\
& a_{2}=\frac{\left(t_{i+1}-t_{i}\right)^{2}}{2} \ddot{\gamma}\left(t_{i}\right)\left(1+O\left(\frac{1}{m}\right)\right)+O\left(\frac{1}{m^{2}}\right) \dot{\gamma}\left(t_{i}\right)+O\left(\frac{1}{m^{3}}\right) .
\end{aligned}
$$

Proof. From (5),

$$
\left(\alpha^{2}, \alpha-\alpha^{2}\right)=\frac{\left(t_{i+2}-t_{i}\right)\left(t_{i+2}-t_{i}, t_{i+1}-t_{i+2}\right)}{\left(t_{i+1}-t_{i}\right)^{2}}+O\left(\frac{1}{m}\right) .
$$


Then by (6),

$$
\begin{aligned}
a_{1}= & \frac{\left(t_{i+1}-t_{i}\right)^{2}\left(p_{\alpha}-\alpha^{2} p_{1}\right)}{\left(t_{i+2}-t_{i}\right)\left(t_{i+1}-t_{i+2}\right)}\left(1+O\left(\frac{1}{m}\right)\right) \\
= & \frac{\left.\left(t_{i+1}-t_{i}\right)^{2}\left(q_{i+2}-q_{i}\right)-\left(\left(t_{i+2}-t_{i}\right)^{2}+O\left(\frac{1}{m^{3}}\right)\right)\left(q_{i+1}-q_{i}\right)\right)}{\left(t_{i+2}-t_{i}\right)\left(t_{i+1}-t_{i+2}\right)}\left(1+O\left(\frac{1}{m}\right)\right) \\
= & \frac{\left(t_{i+1}-t_{i}\right)^{2}\left(\left(t_{i+2}-t_{i}\right) \dot{\gamma}+\left(t_{i+2}-t_{i}\right)^{2} \frac{\ddot{\gamma}}{2}\right)-\left(t_{i+2}-t_{i}\right)^{2}\left(\left(t_{i+1}-t_{i}\right) \dot{\gamma}+\left(t_{i+1}-t_{i}\right)^{2} \frac{\ddot{\gamma}}{2}\right)}{\left(t_{i+2}-t_{i}\right)\left(t_{i+1}-t_{i+2}\right)} \\
& +O\left(\frac{1}{m^{3}}\right) \frac{\left(t_{i+1}-t_{i}\right) \dot{\gamma}+\left(t_{i+1}-t_{i}\right)^{2} \frac{\ddot{\gamma}}{2}+O\left(\frac{1}{m^{3}}\right)}{\left(t_{i+2}-t_{i}\right)\left(t_{i+1}-t_{i+2}\right)} \\
= & \left(t_{i+1}-t_{i}\right) \dot{\gamma}\left(1+O\left(\frac{1}{m}\right)\right)+O\left(\frac{1}{m^{2}}\right) \dot{\gamma}+O\left(\frac{1}{m^{3}}\right),
\end{aligned}
$$

where $\dot{\gamma}, \ddot{\gamma}$ are evaluated at $t_{i}$. In similar fashion,

$$
\begin{aligned}
a_{2} & =\frac{\left(t_{i+1}-t_{i}\right)^{2}\left(\alpha p_{1}-p_{\alpha}\right)}{\left(t_{i+2}-t_{i}\right)\left(t_{i+1}-t_{i+2}\right)}\left(1+O\left(\frac{1}{m}\right)\right) \\
& =\frac{\left(\left(t_{i+1}-t_{i}\right)\left(t_{i+2}-t_{i}\right)+O\left(\frac{1}{m^{3}}\right)\right)\left(q_{i+1}-q_{i}\right)-\left(t_{i+1}-t_{i}\right)^{2}\left(q_{i+2}-q_{i}\right)}{\left(t_{i+2}-t_{i}\right)\left(t_{i+1}-t_{i+2}\right)}\left(1+O\left(\frac{1}{m}\right)\right) \\
& =\frac{\left(t_{i+1}-t_{i}\right)\left(t_{i+2}-t_{i}\right)\left(q_{i+1}-q_{i}\right)-\left(t_{i+1}-t_{i}\right)^{2}\left(q_{i+2}-q_{i}\right)}{\left(t_{i+2}-t_{i}\right)\left(t_{i+1}-t_{i+2}\right)}\left(1+O\left(\frac{1}{m}\right)\right) \\
& +O\left(\frac{1}{m^{2}}\right) \dot{\gamma}+O\left(\frac{1}{m^{3}}\right) \\
& =\frac{\left(t_{i+1}-t_{i}\right)^{2}\left(\left(t_{i+1}-t_{i}\right)-\left(t_{i+2}-t_{i}\right)\right) \ddot{\gamma}}{2\left(t_{i+1}-t_{i+2}\right)}\left(1+O\left(\frac{1}{m}\right)\right)+O\left(\frac{1}{m^{2}}\right) \dot{\gamma}+O\left(\frac{1}{m^{3}}\right) .
\end{aligned}
$$

In particular,

$$
\frac{d Q^{i}}{d s}=O\left(\frac{1}{m}\right) \quad \text { and } \quad \frac{d^{2} Q^{i}}{d s^{2}}=O\left(\frac{1}{m^{2}}\right),
$$

for $s \in[0, \beta]$. The quadratics $Q^{i}$, determined by $\mathcal{Q}$ and $i$, need to be reparameterized for comparison with the original curve $\gamma$.

3. Proof of Theorem 1. Let $\psi:\left[t_{i}, t_{i+3}\right] \rightarrow[0, \beta]$ be the cubic given by

$$
\psi\left(t_{i}\right)=0, \quad \psi\left(t_{i+1}\right)=1, \quad \psi\left(t_{i+2}\right)=\alpha, \quad \psi\left(t_{i+3}\right)=\beta .
$$

Using Lemma 1 (see http://www.cs.uwa.edu.au/ ryszard/4points/, especially for treatment of $O\left(\frac{1}{m^{2}}\right)$ errors in $\left.(5)\right)$ yields

$$
\frac{d^{k} \psi}{d t^{k}}=O(m), \quad \text { for } k=1,2,3
$$


In particular, $\psi$ is a diffeomorphism for $m$ large. Define $\tilde{\gamma}_{i}=Q^{i} \circ \psi:\left[t_{i}, t_{i+3}\right] \rightarrow \mathbb{R}^{2}$. Then $\tilde{\gamma}_{i}$ is a polynomial of degree at most 6 and, using (7), (8), it turns out that its derivatives of all orders are $O(1)$. The $C^{4}$ function $f=\tilde{\gamma}_{i}-\gamma$ is $\mathbf{0}$ at $t_{i}, t_{i+1}, t_{i+2}, t_{i+3}$, and consequently,

$$
f(t)=\left(t-t_{i}\right)\left(t-t_{i+1}\right)\left(t-t_{i+2}\right) g(t), \quad \text { where } g(t)=\left(t-t_{i+3}\right) h(t),
$$

and $g, h:\left[t_{i}, t_{i+3}\right] \rightarrow \mathbb{R}^{2}$ are $C^{1}, C^{0}$ respectively. Here we use Lemma 2.1 of Part I of [5], and to estimate errors. Because $\frac{d^{4} f}{d t^{4}}=O(1), h=O(1)$. Therefore, $g=O\left(\frac{1}{m}\right)$. Also, $\dot{g}=O\left(\frac{d^{4} f}{d t^{4}}\right)=O(1)$. Therefore,

$$
\dot{f}=O\left(\frac{1}{m^{3}}\right) \quad \text { and } \quad f=O\left(\frac{1}{m^{4}}\right) .
$$

Write $\dot{\tilde{\gamma}}_{i}(t)$ in the form $(1+\langle\dot{f}(t), \dot{\gamma}(t)\rangle) \dot{\gamma}(t)+v(t)$, where $v(t)$ is the projection of $\dot{f}(t)$ onto the line orthogonal to $\dot{\gamma}(t)$. By $(9), v=O\left(\frac{1}{m^{3}}\right)$ and, because $\|\dot{\gamma}\|=1$,

$$
\left\|\dot{\tilde{\gamma}}_{i}(t)\right\|=(1+\langle\dot{f}(t), \dot{\gamma}(t)\rangle)\|\dot{\gamma}(t)\|+O\left(\frac{1}{m^{6}}\right) .
$$

Then

$$
\begin{aligned}
\int_{t_{i}}^{t_{i+3}}\left\|\dot{\tilde{\gamma}}_{i}(t)\right\|-\|\dot{\gamma}(t)\| d t & =\int_{t_{i}}^{t_{i+3}}\langle\dot{f}(t), \dot{\gamma}(t)\rangle d t+O\left(\frac{1}{m^{7}}\right) \\
& =-\int_{t_{i}}^{t_{i+3}}\langle f(t), \ddot{\gamma}(t)\rangle d t+O\left(\frac{1}{m^{7}}\right),
\end{aligned}
$$

after integration by parts. By (9), the right-hand side is $O\left(\frac{1}{m^{5}}\right)$; namely,

$$
\int_{t_{i}}^{t_{i+3}}\|\dot{\gamma}(t)\| d t-d\left(Q^{i}\right)=O\left(\frac{1}{m^{5}}\right),
$$

and so

$$
\tilde{d}(\mathcal{Q}) \equiv \sum_{j=0}^{\frac{m}{3}-1} d\left(Q^{3 j}\right)=d(\gamma)+O\left(\frac{1}{m^{4}}\right) .
$$

Notice that a track-sum $\tilde{\gamma}$ of the arcs swept out by the $Q^{3 j}$ gives an $O\left(\frac{1}{m^{4}}\right)$ uniformly accurate approximation of the image of $\gamma$. Although $\tilde{\gamma}$ is not $C^{1}$ at $t_{3}, t_{6}, \ldots, t_{m-3}$, the differences in left and right derivatives are $O\left(\frac{1}{m^{3}}\right)$, and hardly discernible when $m$ is large.

4. Convergence rates. In Example 3, piecewise 3-point quadratic interpolation gives a poor estimate of the semicircle, in particular of its length. Now we check the performance of the alternative piecewise 4-point quadratic interpolation used in the proof of Theorem 1.

EXAMPLE 6. As in Example 3, take $m=6$ and use the same more-or-less uniform sampling of parameters $t_{i}$. The piecewise 4-point quadratic interpolant in Fig. 4 is more semicircular and the error in the length estimate is reduced from 0.0601 to -0.0072 .

Let $\epsilon_{m}$ be the absolute value of the error in the length estimate using piecewise 4-point quadratic interpolation, where values of $m$ not divisible by 3 are accounted for 


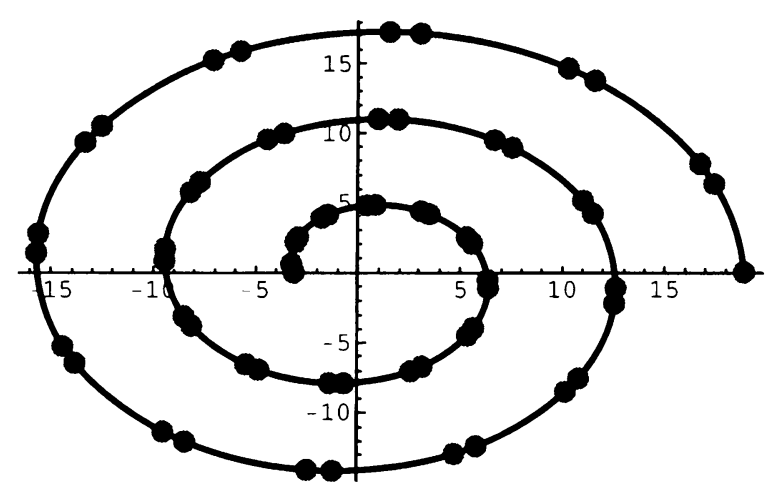

FIG. 3. A piecewise 4-point quadratic approximation to a spiral (singular point excluded), using the more-or-less uniform sampling of Example 3 and 61 data points $(m=60)$. True length: 173.608, estimate: 173.539 , piecewise 3-point quadratic estimate: 181.311.

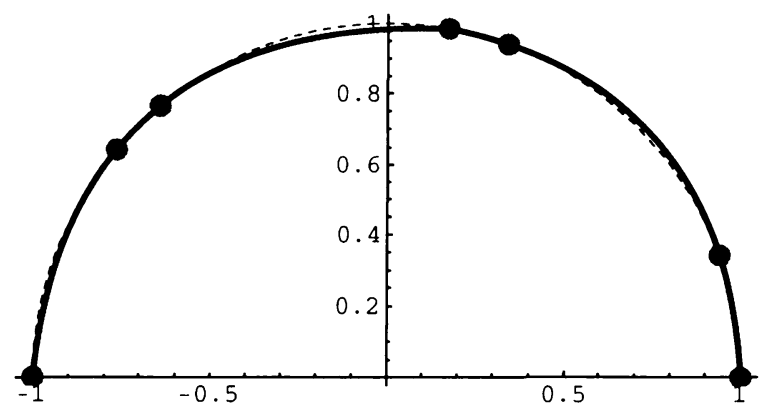

FIG. 4. Piecewise 4-point quadratic using 7 data points $(m=6)$ from a semicircle (shown dashed). Length estimate: $\pi-0.00723637$.

by a simple modification. The plot in Fig. 5 of $-\log \epsilon_{m}$ against $\log m$ for $3 \leq m \leq 100$ appears linear, and the least-squares estimate of slope is approximately 3.83. According to Theorem 1, the limiting slope is at least 4 as $m \rightarrow \infty$. So the evidence points to exactly quartic convergence in this example

Our experiences with other curves and other more-or-less uniform samplings are similar to Example 6. We have also mentioned the spiral in connection with the sampling of Example 3. We give one further example, of a cubic. There are not a lot of changes.

EXAMPLE 7. The cubic is given parametrically by $\gamma(t)=\left(t,-t^{3}\right)$ for $t \in[0.1,0.5]$ and sampled in the random fashion of Example 4 for $3 \leq m \leq 100$. The plot of $-\log \epsilon_{m}$ against $\log m$ is shown in Fig. 6. The least-squares estimate of slope is 4.00 . So the evidence suggests only quartic convergence.

5. Concluding remarks. The condition that sampling be more-or-less uniform is rather unrestrictive. For $\epsilon>0$, sampling is said to be $\epsilon$-uniform when, in some parameterization, 


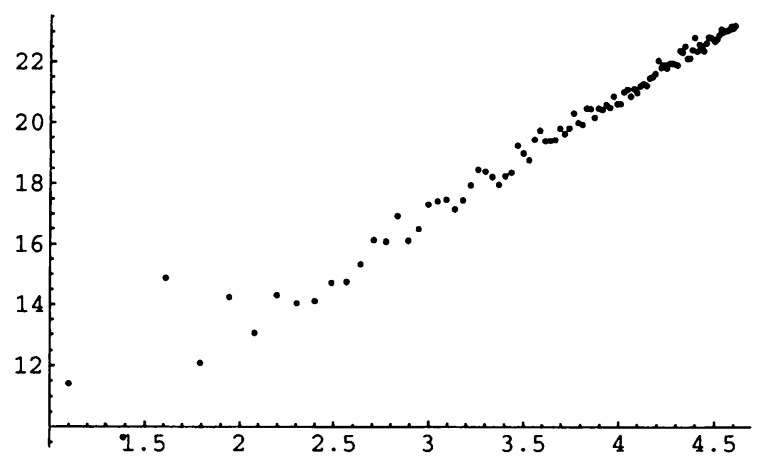

FIG. 5. $-\log \epsilon_{m}$ against $\log m$, for the semicircle and $3 \leq m \leq 100$. Estimate of slope: 3.82694 .

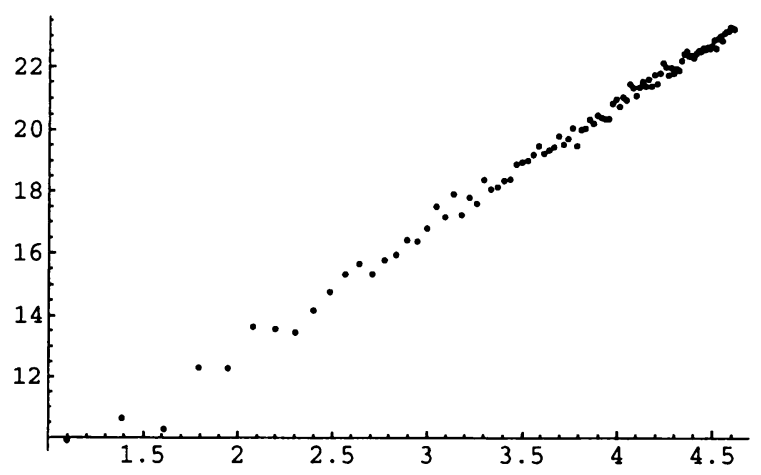

FIG. 6. $-\log \epsilon_{m}$ against $\log m$, for the cubic and $3 \leq m \leq 100$.

Estimate of slope: 4.00347 .

$$
t_{i}=\frac{i T}{m}+O\left(\frac{1}{m^{1+\epsilon}}\right) .
$$

For this more regular kind of sampling, it is possible to get results like Theorem 1 using simpler constructions for $\tilde{d}(\mathcal{Q})$, as in [7], [8].

There is also some analogous work for estimating lengths of digitized curves; indeed, the analysis of digitized curves in $\mathbb{R}^{2}$ is one of the most intensively studied subjects in image data analysis. A digitized curve is the result of a process (such as contour tracing, $2 D$ skeleton extraction, or $2 D$ thinning) that maps a curve-like object (such as the boundary of a region) onto a computer-representable curve. As before, $\gamma:[0, T] \rightarrow \mathbb{R}^{2}$ is a strictly convex curve parameterized by arc-length. An analytical description of $\gamma$ is not given, and numerical measurements of points on $\gamma$ are corrupted by a process of digitization: $\gamma$ is digitized within an orthogonal grid of points $\left(\frac{i}{m}, \frac{j}{m}\right)$, where $i, j$ are permitted to range over integer values, and $m$ is a fixed positive integer called the grid resolution. Depending on the digitization model [2], $\gamma$ is mapped onto a digital curve and approximated by a polygon $\hat{\gamma}_{m}$ whose length is an estimator for $d(\gamma)$. Approximating polygons $\hat{\gamma}_{m}$ based on 
local configurations of digital curves does not ensure multigrid length convergence, but global approximation techniques yield linearly convergent estimates, namely,

$$
d(\gamma)-d\left(\hat{\gamma}_{m}\right)=O\left(\frac{1}{m}\right)
$$

$[3,9]$. In the special case of discrete straight line segments in $\mathbb{R}^{2}$, a stronger result is proved [1], where superlinear $O\left(\frac{1}{m^{1,5}}\right)$ orders of asymptotic length estimates are given. In Theorem 1, convergence is of order 4 , but $\mathcal{Q}$ arises from more-or-less uniform sampling, as opposed to digitization. So strict comparisons cannot yet be made. These issues will be revisited in the future.

6. Acknowledgment. This research was supported by an Australian Research Council Small Grant. The authors acknowledge with thanks several stimulating conversations with Professor R. Klette on matters related to this paper.

\section{REFERENCES}

[1] L. Dorst and A. W. M. Smeulders, Discrete straight line segments: Parameters, primitives and properties, In: R. Melter, P. Bhattacharya, and A. Rosenfled (eds.), Contemp. Math., Vol. 119, Amer. Math. Soc., Providence, RI, 1991, pp. 45-62

[2] R. Klette, Approximation and representation of $3 D$ objects, In: R. Klette, A. Rosenfeld, and F. Sloboda (eds.), Advances in Digital and Computational Geometry, Springer, Singapore, 1998, pp. 161-194

[3] R. Klette, V. Kovalevsky, and B. Yip, On the length estimation of digital curves, In: L. J. Latecki, R. A. Melter, D. M. Mount, and A. Y. Wu (eds.), SPIE Conference Proceedings Vision Geometry VIII, Denver, Colorado, 1999, pp. 52-63

[4] R. Klette, A. Rosenfeld, and F. Sloboda (eds.), Advances of Digital and Computational Geometry, Springer-Verlag, 1998

[5] J. Milnor, Morse Theory, Annals. of Math. Studies, Vol. 51, Princeton University Press, Princeton, NJ, 1963

[6] P. A. P. Moran, Measuring the length of a curve, Biometrika 53, 359-364 (1966)

[7] L. Noakes, R. Kozera, and R. Klette, Length estimation for curves with different samplings, In: G. Bertrand, A. Imiya, and R. Klette (eds.), Digital and Image Geometry, Lecture Notes in Computer Science, Vol. 2243, Springer-Verlag, Berlin Heidelberg, 2001 pp. 339-351

[8] L. Noakes, R. Kozera, and R. Klette, Length estimation for curves with $\epsilon$-uniform sampling, In: W. Skarbek (ed.), Proceedings of 9th International Conference on Computer Analysis of Images and Patterns, Warsaw, Poland, 2001. Lecture Notes in Computer Science, Vol. 2124, Springer-Verlag, Berlin Heidelberg, pp. 518-528

[9] F. Sloboda, B. Zaťko, and J. Stoer, On approximation of planar one-dimensional continua, In: R. Klette, A. Rosenfeld, and F. Sloboda (eds.), Advances in Digital and Computational Geometry, Springer, Singapore, 1998, pp. 113-160

[10] H. Steinhaus, Praxis der Rektifikation und zum Längenbegriff, Akad. Wiss. Leipzig. Berlin 82, 120-130 (1930)

[11] B. L. van der Waerden, Geometry and Algebra in Ancient Civilizations, Springer-Verlag, Berlin, 1983

[12] A. G. Werschulz and H. Woźniakowski, What is the complexity of surface integration?, J. Complexity 17, 442-466 (2001)

[13] K. Mørken and K. Scherer, A general framework for high-accuracy parametric interpolation, Math. of Comp. 66, No. 217, 237-260 (1997) 